\title{
EFEITO DE NÍVEIS CRESCENTES DE CALAGEM NO CRESCIMENTO E ESTADO NUTRICIONAL DE FEDEGOSO ${ }^{1}$
}

\author{
EDUARDO A. L. ERASMO ${ }^{2}$, SILVANO BIANCO ${ }^{3}$, ROBINSON A. PITELLI ${ }^{4}$ e PAULO BERRINGELI ${ }^{5}$
}

\section{RESUMO}

A distribuição e desenvolvimento de plantas, a exemplo daquelas que infestam as áreas agrícolas, é influenciada por diversos fatores, a como aqueles ligados às caraterísticas do solo, naturais ou impostas pelo homem. Assim, o presente trabalho de pesquisa foi desenvolvido em condições de casa-de-vegetação com o objetivo de estudar o efeito de níveis crescentes de calagem no crescimento e estado nutricional de Senna obtusifolia, planta daninha comum em ecossistemas agrícolas. $\mathrm{O}$ delineamento experimental adotado foi o inteiramente casualizado, com seis tratamentos e quatro repetições. Os tratamentos constituíram-se em quantidades de calcário dolomítico calcinado com $38 \%$ de $\mathrm{CaO}$ e $20 \%$ de $\mathrm{MgO}$, com um poder de neutralização total (PRNT) de 104\%: 0, 2, 4, 6, 8 e $10 \mathrm{t} / \mathrm{ha}$. As parcelas experimentais constituíram-se de recipientes de plástico com capacidade de 5 litros, preenchidos com terra coletada na camada arável de um latossolo vermelho escuro distrófico, "A" moderado, textura média. Após um período de crescimento de 56 dias as plantas foram coletadas e separadas em seus componentes vegetativos, sendo colocados a secar em estufa de circulação forçada de ar $\left(70^{\circ} \mathrm{C}\right.$ por 72 horas) para determinação do peso seco. O material foi pesado e moído e analisado a nível de composição de nutrientes. Os resultados mostraram efeitos negativos dos níveis crescentes de calcário, sobre o crescimento das plantas de S. obtusifolia, evidenciando-se decréscimos nos valores da matéria seca acumulada a partir da dose de $4 \mathrm{t} / \mathrm{ha}$ de calcário ( $\mathrm{pH} 5,8$ ), com efeitos mais marcantes nas raízes. A aplicação de doses crescentes de calcário promoveu um aumento progressivo nos teores de $\mathrm{Ca}$ e $\mathrm{Mg}$ no solo, o que talvez possa ter estimulado a competição entre cátions, reduzindo a absorção do K. Os elementos $\mathrm{N}$ e $\mathrm{P}$ foram os mais absorvidos pela planta.

Palavras chave: Calagem, nutrição, $\mathrm{pH}$, Senna obtusifolia.

\section{ABSTRACT \\ Effect of growing levels of liming in the growth nutricional of Senna obtusifolia}

The distribution and development of plants, to example of those that infest the agricultural areas, the is influenced several factors, exemply those tied the caraterísticas of the natural soil or imposed by the man. Like this being, the present research work objectified, to study the effect of growing levels of liming in the growth and state nutritional of Senna obtusifolia, it weed common in agricultural ecosystems. For this, the present research was developed, in conditions of Green House belonging to the Department of Applied Biology to the Agricultural, FCAVJ-UNESP, under randomized completely, with six treatments and four replications. The treatments were constituted in the amounts of liming: 00, 02, 04, 06, 08 and

\footnotetext{
${ }^{1}$ Recebido para publicação em 7/7/99 e na forma revisada em 15/2/2000.

${ }^{2}$ Prof $^{\circ}$ Dr., Fundação Universidade do Tocantins. Av. Madrid Q. 6, Lt. 8 e 9, Jardim Sevilha, CEP: 77410-470.

${ }^{3}$ Prof $^{\circ}$ Dr., Dept ${ }^{\circ}$ de Biologia Aplicada à Agropecuária, FCAVJ/UNESP. CEP: 14870-000, Jaboticabal/SP, Brasil.

${ }_{5}^{4}$ Prof $^{\mathrm{O}}$ Dr., Dept ${ }^{\mathrm{O}}$ de Biologia Aplicada à Agropecuária, FCAVJ/UNESP. CEP: 14870-000, Jaboticabal/SP, Brasil.

${ }^{5}$ Prof $^{\circ}$ Dr., Dept ${ }^{\circ}$ de Tecnologia de Alimentos, FCAVJ/UNESP. CEP: 14870-000, Jaboticabal/SP, Brasil.
} 
$10 \mathrm{t} /$ ha, being the liming used the dolomítico roasted with $38 \%$ of $\mathrm{Ca}$ and $20 \%$ of $\mathrm{MgO}$, with a relative power of total neutralization (PRNT) of $104 \%$. The experimental portions had been constituted of pots of plastic with capacity of 5 litter filled with earth collected in the layer arável of a Latossolo Red Dark Distrófico, the moderate, medium texture. After a period of growth of 56 days the plants were collected and separated in its vegetative components, being placed to dry in stove of forced circulation of air $\left(70^{\circ} \mathrm{C}\right.$ for 72 hours) for determination of the dry weight. After heavy, the material was triturated and analyzed the composition level of nutritions. The results for

\section{INTRODUÇÃO}

Dentro dos fatores determinantes no processo de colonização e estabelecimento das plantas em determinado ambiente, as características químicas do solo desempenham um papel predominante. Assim, a disponibilidade de um ou vários nutrientes específicos, bem como o $\mathrm{pH}$ do solo, podem influenciar na importância relativa de determinadas espécies de plantas daninhas num agroecossistema específico. Andreasen et al. (1991), estudando a correlação entre algumas propriedades do solo e a distribuição de espécies daninhas em solos agrícolas, concluíram que o tipo de cultura e o conteúdo de argila no solo foram as variáveis mais determinantes, mas outros fatores, como $\mathrm{pH}$ e concentração de nutrientes no solo, também influenciaram em alto grau.

Em algumas plantas daninhas o pH, além de influenciar a sua distribuição, afeta a sua habilidade competitiva, seja pela limitação da disponibilidade de algum nutriente, ou por afetar diretamente seu crescimento, principalmente das raízes, ocasionando uma menor exploração do meio. Weaver \& Hamill (1985), estudando o efeito do $\mathrm{pH}$ na habilidade competitiva e conteúdo de nutrientes nas folhas de plantas de milho e três espécies daninhas, constataram que em todas as faixas de $\mathrm{pH}$ avaliado $(4,8 ; 6,0$ e 7,3) as porcentagens de $\mathrm{N}$ e $\mathrm{K}$ nos tecidos foliares das now obtained they evidenced negative effects of the growing levels of liming, on the growth of the plants of $\mathrm{S}$. obtusifolia, being evidenced reductions in the values of the dry matter accumulated starting from the dose of $4 \mathrm{t} / \mathrm{ha}$ of calcareous ( $\mathrm{pH} 5,8)$, with effects more marcantes in the roots. The application of growing doses of calcareous provoked in the soil a progressive increase in the texts of $\mathrm{Ca}$ and $\mathrm{Mg}$, what perhaps can have stimulated the competition among cátions, reducing the absorption of $\mathrm{K}$. The elements $\mathrm{N}$ and $\mathrm{P}$ was the more absorbed by the plant.

Key words: Weed, nutrition, $\mathrm{pH}$.

plantas daninhas foram superiores às do milho. Ramakrishnan \& Gupta (1973) observaram grandes diferenças em plantas de Cynodon dactylon provenientes de solos diferentes, onde verificaram que plantas originárias de solos calcários apresentaram melhor crescimento do que aquelas provenientes de outros solos, quando desenvolvidas em teores baixos de $\mathrm{N}$ e $\mathrm{K}$.

Variação de tolerância entre espécies de plantas daninhas são evidentes, conforme constataram Buchanan et al. (1975) os quais, utilizando níveis de $\mathrm{pH}$ variando de 4,7 até 6,3, verificaram que algumas espécies foram altamente tolerantes a $\mathrm{pH}$ baixo, tais como Crotalaria spectabilis Roth., Cassia occidentalis e Digitaria sanguinalis, enquanto outras como: Desmodium tortuosum, Amaranthus retroflexus, Stellaria media, Taraxacum officinale e Brassica kaper foram severamente prejudicadas. No entanto, outras plantas possuem uma grande elasticidade no seu crescimento a diferentes $\mathrm{pH}$ do meio, a exemplo de Cenchrus echinatus, onde o seu padrão de alocação de matéria seca e nutrientes não varia quando as plantas são submetidas a pH variando de 3,5 a 7,0 (Pavani,1992). A planta daninha Senna obtusifolia pode ser considerada uma planta que tolera uma ampla variação de $\mathrm{pH}$ do solo, crescendo normalmente em $\mathrm{pH}$ que varia de 3,2 até 7,9 (Creel et al., 1968 e Teem et al., 1974). 
Neste sentido, o presente trabalho teve por objetivo estudar o crescimento e acúmulo de nutrientes de Senna obtusifolia, quando crescida sob diferentes doses de calcário aplicados ao solo.

\section{MATERIAL E MÉTODOS}

O trabalho foi instalado no dia 10/3/1994 e conduzido em condições de Casa-de-Vegetação pertencente ao Departamento de Biologia Aplicada a Agropecuária, da Faculdade de Ciências Agrárias e Veterinárias da UNESP, Campus de Jaboticabal-SP.

Para o crescimento das plantas foram utilizados recipientes de plástico com capacidade de 5 litros, preenchidos com terra coletada na camada arável de um Latossolo Vermelho Escuro Distrófico, A moderado, textura média. A terra foi seca à sombra e peneirada em tamis de malha de $2 \mathrm{~mm}$ sendo suas principais características químicas e físicas apresentadas nas Tabelas 1 e 2, respectivamente.

O delineamento experimental adotado foi inteiramente casualizado, com seis tratamentos e quatro repetições. Os tratamentos constituíram-se nas quantidades de calcário a saber: 00, 02, 04, 06, 08 e 10 t/ha. O calcário utilizado foi o dolomítico calcinado com $38 \%$ de $\mathrm{CaO}$ e $20 \%$ de $\mathrm{MgO}$, com um poder relativo de neutralização total (PRNT) de $104 \%$. O período experimental foi de 56 dias após a emergência das plântulas de S. obtusifolia.

A mistura do calcário com o substrato foi efetuada em sacos de plástico de dez litros. Para tanto, o calcário e a terra foram colocados no saco plástico que, após ter sido hermeticamente fechado, foi agitado intensamente até ser conseguida uma mistura homogênea do corretivo no substrato. A seguir, o conteúdo foi sendo paulatinamente umedecido e agitado, até que a umidade atingisse $70 \%$ do poder de embebição $(39.9 \% \mathrm{p} / \mathrm{p})$. O período de incubação para a reação do solo foi de quinze dias, havendo revolvimento diário do substrato e manutenção da umidade, através das pesagens e reposições da água evaporada.

Após o período de incubação de vinte dias, foi realizada a semeadura depositando-se vinte sementes sem dormência em cada vaso. Para a quebra de dormência as sementes foram previamente tratadas com $\mathrm{H}_{2} \mathrm{SO}_{4}$ por um período de 15 minutos, após o qual foram lavadas em água corrente, por igual período de tempo. Quinze dias após a emergência, foi realizado um desbaste, deixando-se uma planta por vaso.

No momento da semeadura, conforme análise química do solo, realizou-se uma adubação em todos os vasos com 200 ppm de P e $150 \mathrm{ppm}$ de $\mathrm{K}$, utilizando-se como fontes super fosfato simples e cloreto de potássio respectivamente, segundo recomendações para a cultura da soja.

Aos 56 dias após a emergência foi efetuada a avaliação final do ensaio. Para tanto, as plantas foram cortadas na altura do colo e separadas em seus componentes vegetativos e reprodutivos. As raízes foram separadas do solo através da lavagem sobre peneira de $2 \mathrm{~mm}$, utilizando-se jatos de água. Todas as partes da planta foram lavadas (por imersão) inicialmente em água de torneira, depois água destilada e, finalmente, com água deionizada. O material foi seco em estufa de circulação forçada de ar $\left(70^{\circ} \mathrm{C}\right.$ por 72 horas) e depois foi determinado o peso seco através de balança eletrônica com precisão de centésimos. Depois de pesado, o material foi moído em micromoinho tipo "Wiley", malha vinte, acondicionado em vidros previamente identificados e armazenado em local seco, para posterior análise.

A análise do $\mathrm{N}$ total foi efetuada por metodologia do semi-microkjeldahl e a do P pela metodologia do ácido fosfovanadato-molibdico, descrita por Sarruge \& Haag (1974). Para a extração do $\mathrm{K}, \mathrm{Ca}$ e $\mathrm{Mg}$ foi utilizado o método descrito por Jorgensen (1977), através de espectrofotometria de absorção atômica. 
TABELA 1. Principais características químicas da terra utilizada como substrato, no ensaio para avaliação da calagem do substrato sobre S. obtusifolia.

\begin{tabular}{|c|c|c|c|c|c|c|c|c|c|}
\hline \multirow{2}{*}{$\begin{array}{l}\text { M.O. } \\
\text { G/kg }\end{array}$} & \multirow{2}{*}{$\begin{array}{c}\mathrm{pH} \\
\left(\mathrm{CaCl}_{2}\right)\end{array}$} & \multirow{2}{*}{$\begin{array}{l}\text { P resina } \\
\mu \mathrm{g} / \mathrm{cm}^{3}\end{array}$} & \multicolumn{6}{|c|}{$\mathrm{Cmol}_{\mathrm{d} \mathrm{dm}^{3}}$} & \multirow{2}{*}{$\begin{array}{l}\mathrm{V} \\
\%\end{array}$} \\
\hline & & & $\mathrm{K}^{+}$ & $\mathrm{Ca}^{+2}$ & $\mathrm{Mg}^{+2}$ & $\mathrm{H}+\mathrm{Al}$ & SB & $\mathrm{T}$ & \\
\hline 2,4 & 3,7 & 5 & 0,1 & 0,5 & 0,2 & 8,0 & 0,8 & 8,8 & 9 \\
\hline
\end{tabular}

TABELA 2. Principais características físicas da terra utilizada como substrato.

\begin{tabular}{ccccc}
\hline Argila (\%) & Limo (\%) & Areia Fina (\%) & Areia Grossa (\%) & Classe Textural \\
\hline 26,0 & 3,0 & 33,0 & 38,0 & franco-argilo-arenosa \\
\hline
\end{tabular}

No final do experimento também foram retiradas amostras do substrato para análises das características químicas. Os dados foram submetidos a estudos de regressão polinomial no parâmetro teores de nutrientes nas plantas e teste de Tukey para a comparação de médias no caso específico de acúmulos de matéria seca e nutrientes. Foram realizadas correlações entre a concentração de nutrientes no solo e na planta, bem como algumas relações, com o acúmulo de matéria seca de plantas, sendo as médias comparadas pelo teste $\mathrm{t}$ ao nível $1 \%$ de probabilidade.

\section{RESULTADOS E DISCUSSÃO}

Na Tabela 3 estão apresentados os resultados referentes às análises das principais características químicas dos substratos dos vasos no final do período experimental (56 dias após a semeadura de $S$. obtusifolia). Os teores de matéria orgânica e K no solo não foram influenciados com a adição de calcário. Com o aumento das doses de calcário, os valores do $\mathrm{pH}$ e as concentrações de $\mathrm{P}$, Ca e de $\mathrm{Mg}$ no substrato foram incrementados enquanto os teores de $\mathrm{H}+\mathrm{Al}$ decresceram, estabilizando-se a partir do tratamento de 6 t/ha de calcário. Tomando-se como referência a $5^{\text {a }}$ aproximação de recomendações de corretivos e fertilizantes para o estado de Goiás (UFG/EMGOPA, 1988) os teores de nutrientes no solo podem ser considerados médios para o $\mathrm{K}$; muito alto para o $\mathrm{P}$ e altos para o Ca e Mg nos tratamentos acima de 4 t/ha de calcário.

Os resultados referentes às relações entre os elementos $\mathrm{Ca}, \mathrm{Mg}$ e $\mathrm{K}$ no substrato e às suas participações na capacidade de troca catiônica estimada estão apresentados na Tabela 4. A medida em que a dose de calcário foi elevada, houve aumento nas relações $\mathrm{Ca} / \mathrm{K}$ e $\mathrm{Mg} / \mathrm{K}$ e decréscimo na relação $\mathrm{Ca} / \mathrm{Mg}$. O Ca e o $\mathrm{Mg}$ tiveram maior participação na CTC estimada, enquanto que a participação do $\mathrm{K}$ não foi alterada. $\mathrm{O}$ aumento na participação do $\mathrm{Mg}$ foi mais acentuada que do $\mathrm{Ca}$, provavelmente em decorrência da composição do calcário utilizado. Estes resultados referentes às análises químicas do substrato não serão discutidos em si, visto que os efeitos da aplicação de calcário sobre as propriedades do solo são amplamente conhecidos e bem documentados. No entanto, serão úteis para explicar os resultados relativos ao crescimento e a nutrição mineral da $S$. obtusifolia.

$\mathrm{Na}$ Tabela 5 estão relacionadas as variações no acúmulo de matéria seca dos diferentes componentes vegetativos de $S$. obtusifolia, aos 56 dias de crescimento, em função de doses crescentes de calcário. 
TABELA 3. Características químicas do substrato aos 56 dias após a emergência de S. obtusifolia, submetidos a doses crescentes de calcário.

\begin{tabular}{ccccccccccc}
\hline $\begin{array}{c}\text { Doses } \\
(\mathrm{t} / \mathrm{ha})\end{array}$ & $\begin{array}{c}\mathrm{M} . \mathrm{O} . \\
(\mathrm{g} / \mathrm{kg})\end{array}$ & $\begin{array}{c}\mathrm{P} \text { resina } \\
(\mathrm{ug} / \mathrm{ml})\end{array}$ & $\begin{array}{c}\mathrm{pH} \\
\left(\mathrm{CaCl}_{2}\right)\end{array}$ & $\mathrm{K}$ & $\mathrm{Ca}$ & $\mathrm{Mg}$ & $\mathrm{H}+\mathrm{Al}$ & $\mathrm{SB}$ & $\mathrm{T}$ & $\begin{array}{c}\mathrm{V} \\
(\%)\end{array}$ \\
\hline & & & & & & & $\mathrm{Cmol}_{\mathrm{c}} / \mathrm{dm}^{3}$ & & & \\
0 & 1,7 & 137 & 4,3 & 0,16 & 0,65 & 0,17 & 4,7 & 1,0 & 5,7 & 17,3 \\
2 & 1,7 & 146 & 5,2 & 0,12 & 1,51 & 0,39 & 2,8 & 2,0 & 4,8 & 41,9 \\
4 & 1,7 & 247 & 5,8 & 0,19 & 2,93 & 0,73 & 2,2 & 3,9 & 6,1 & 63,6 \\
6 & 1,8 & 221 & 6,1 & 0,17 & 2,80 & 1,57 & 1,8 & 4,5 & 6,3 & 71,6 \\
8 & 1,7 & 307 & 6,1 & 0,18 & 3,47 & 1,35 & 1,9 & 5,0 & 6,9 & 72,5 \\
10 & 1,8 & 221 & 6,5 & 0,15 & 3,25 & 2,13 & 1,6 & 5,5, & 7,1 & 77,6 \\
\hline
\end{tabular}

TABELA 4. Efeito das doses crescentes de calcário nas relações $\mathrm{Ca} / \mathrm{Mg}, \mathrm{Ca} / \mathrm{K}, \mathrm{Mg} / \mathrm{K} \mathrm{e} \mathrm{K} /(\mathrm{Ca}+\mathrm{Mg})$ no solo, e na porcentagem de saturação de $\mathrm{Ca}, \mathrm{Mg}$ e $\mathrm{K}$ em relação ao valor $\mathrm{T}$ do solo aos 56 dias após a emergência das plantas.

\begin{tabular}{cccccccc}
\hline $\begin{array}{c}\text { Doses } \\
(\mathrm{g} / \mathrm{ha})\end{array}$ & $\mathrm{Ca} / \mathrm{Mg}$ & $\mathrm{Ca} / \mathrm{K}$ & $\mathrm{Mg} / \mathrm{K}$ & $\mathrm{K} /(\mathrm{Ca}+\mathrm{Mg})$ & $\mathrm{Ca}$ & $\mathrm{Mg}$ & $\mathrm{K}$ \\
\cline { 6 - 8 } & & & & & & $\%$ de $\mathrm{T}$ & \\
\hline 0 & 3,83 & 4,06 & 1,06 & 0,195 & 11,40 & 2,98 & 2,81 \\
4 & 3,87 & 12,58 & 3,25 & 0,063 & 31,46 & 8,13 & 2,50 \\
6 & 4,01 & 15,42 & 3,84 & 0,052 & 48,03 & 11,97 & 3,11 \\
8 & 1,78 & 16,47 & 9,24 & 0,038 & 44,44 & 24,92 & 2,70 \\
10 & 2,57 & 19,28 & 7,50 & 0,037 & 50,29 & 19,57 & 2,61 \\
\hline
\end{tabular}

TABELA 5. Efeito das doses crescentes de calcário nos peso da matéria seca de plantas (g/vaso) de $S$. obtusifolia aos 56 dias após a emergência.

\begin{tabular}{cccccc}
\hline \multirow{2}{*}{$\begin{array}{c}\text { Doses } \\
\text { (t/ha) }\end{array}$} & Raizes & Folhas & Caule & Inflorescência & Total \\
\cline { 2 - 6 } & $3,62 \mathrm{a}$ & $2,70 \mathrm{ab}$ & $4,32 \mathrm{a}$ & $0,24 \mathrm{~b}$ & $10,86 \mathrm{a}$ \\
0 & $3,39 \mathrm{a}$ & $3,11 \mathrm{a}$ & $4,77 \mathrm{a}$ & $0,34 \mathrm{a}$ & $11,60 \mathrm{a}$ \\
2 & $1,59 \mathrm{c}$ & $1,95 \mathrm{~d}$ & $2,99 \mathrm{~b}$ & $0,19 \mathrm{bc}$ & $6,71 \mathrm{~b}$ \\
4 & $2,02 \mathrm{~b}$ & $2,47 \mathrm{bc}$ & $2,51 \mathrm{bc}$ & $0,21 \mathrm{bc}$ & $7,21 \mathrm{~b}$ \\
6 & $2,05 \mathrm{~b}$ & $2,25 \mathrm{bcd}$ & $1,79 \mathrm{c}$ & $0,14 \mathrm{c}$ & $6,24 \mathrm{~b}$ \\
8 & $2,19 \mathrm{~b}$ & $2,11 \mathrm{~cd}$ & $1,87 \mathrm{c}$ & $0,19 \mathrm{bc}$ & $6,36 \mathrm{~b}$ \\
10 & $77,40^{* *}$ & $16,21^{* *}$ & $58,92^{* *}$ & $14,17^{* *}$ & $73,25^{* *}$ \\
F. & 0,42 & 0,47 & 0,73 & 0,07 & 8,16 \\
D.M.S. & 7,56 & 8,70 & 10,75 & 16,11 & 6,91 \\
C.V. & & & & & \\
\hline
\end{tabular}

${ }^{* * *}$ Significativo ao nível de $1 \%$ de probabilidade.

${ }^{1}$ Médias seguidas da mesma letra não diferem entre si pelo Teste de Tukey ao nível de 5\% de probabilidade. 
As plantas atingiram os maiores acúmulos de matéria seca nas doses mais baixas de calcário (0 e 2 t/ha) não diferindo estatisticamente entre si, com exceção do parâmetro inflorescência doses superiores a $2 \mathrm{t} / \mathrm{ha}$ de calcário ocasionaram uma redução expressiva no peso seco de todos os órgãos vegetativos, alcançando $43 \%$ no peso seco total de plantas na dose de 4 t/ha, não diferindo estatisticamente do peso seco verificado nas doses subsequentes.

As distribuições da matéria seca acumulada nas diferentes partes da planta de $S$. obtusifolia em função das doses de calcário podem ser observadas na Figura 1. Os resultados mostram que o acúmulo de matéria seca nas folhas sofreram efeitos menos acentuados da calagem. A participação das folhas no total da matéria seca acumulada pelas plantas cresceu com o aumento das doses de calcário, enquanto a participação do caule caiu após 4 t/ha. Observa-se que as menores participações das raízes nos tratamentos de 2,4 e 6 t/ha, refletem as doses onde ocorreram as maiores reduções no peso seco de raízes (Tabela 5) e para o caule esta afirmação corresponde a doses superiores de 6 t/ha. Até a dose de $4 \mathrm{t} / \mathrm{ha}$, a participação do caule havia crescido, em detrimento das raízes.

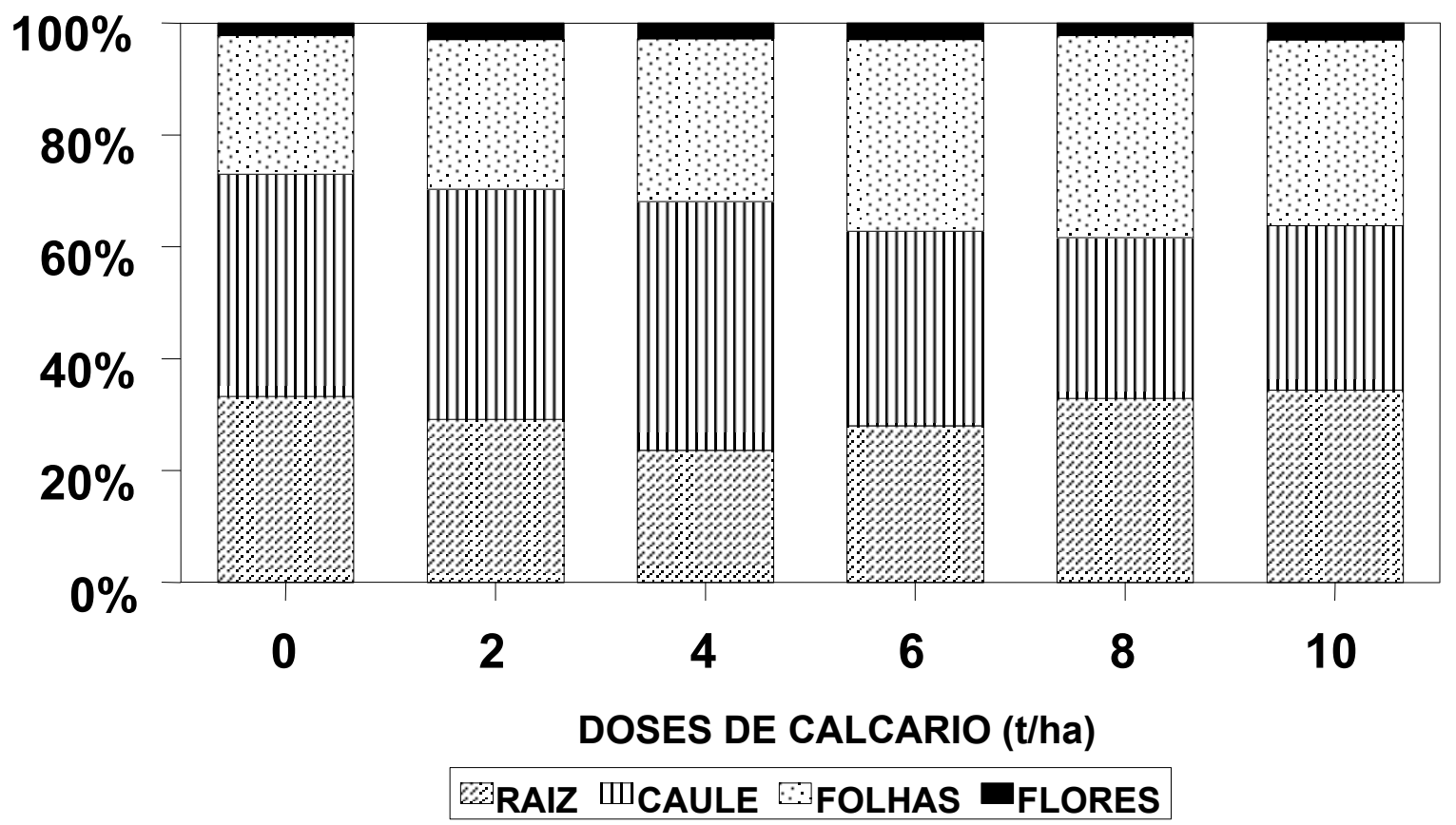

FIGURA 1. Efeito das doses crescentes de calcário na distribuição da matéria seca acumulada nas diferentes partes das plantas de S. obtusifolia aos 56 dias após a emergência.

A representação da concentração de macronutrientes nas plantas de S. obtusifolia em resposta ao incremento de doses de calcário (Figura 2) evidencia um aumento crescente no teor dos nutrientes nas plantas, com a elevação da calagem, tendendo à estabilização nas doses superiores a $6 \mathrm{t} / \mathrm{ha}$. Os maiores incrementos foram constatados para os elementos $\mathrm{N}$ e Mg. Erasmo et al. (1995), estudando curvas de absorção de nutrientes em S. obtusifolia, verificaram aos 60 dias de crescimento teores de: 2,8; 0,21; 2,70; 0,47 e $2,8 \%$ para o $\mathrm{N} ; \mathrm{P} ; \mathrm{K} ; \mathrm{Mg}$ e $\mathrm{Ca}$, respectivamente. Comparando-se estes resultados com os encontrados no presente experimento nas 
doses superiores de 2 t/ha de calcário, observa-se que, apesar de ter havido um crescimento na absorção de nutrientes, as concentrações de $\mathrm{N}$ e K podem ser consideradas baixas, enquanto os teores de $\mathrm{P}$ e $\mathrm{Mg}$ superiores.

$\mathrm{Na}$ Tabela 6 estão descritos os dados referentes aos acúmulos de nutrientes nas plantas de $S$. obtusifolia, aos 56 dias após a emergência, nos diferentes tratamentos experimentais. Os resultados evidenciam que até 4 t/ha de calcário não houve efeito significativo da calagem sobre a absorção do $\mathrm{N}$ do solo, sendo que o incremento constatado nas doses subsequentes não diferiram estatisticamente. Os acúmulos de $\mathrm{P}$ e $\mathrm{Mg}$ foram superiores à testemunha $(0 \mathrm{t} / \mathrm{ha})$ em todas os tratamentos onde foi aplicado calcário, não diferindo estatisticamente entre si. Os acúmulos de $\mathrm{K}$ e Ca foram incrementados com a aplicação de 2 t/ha, decrescendo nas doses subsequentes, como resposta da redução na matéria seca.
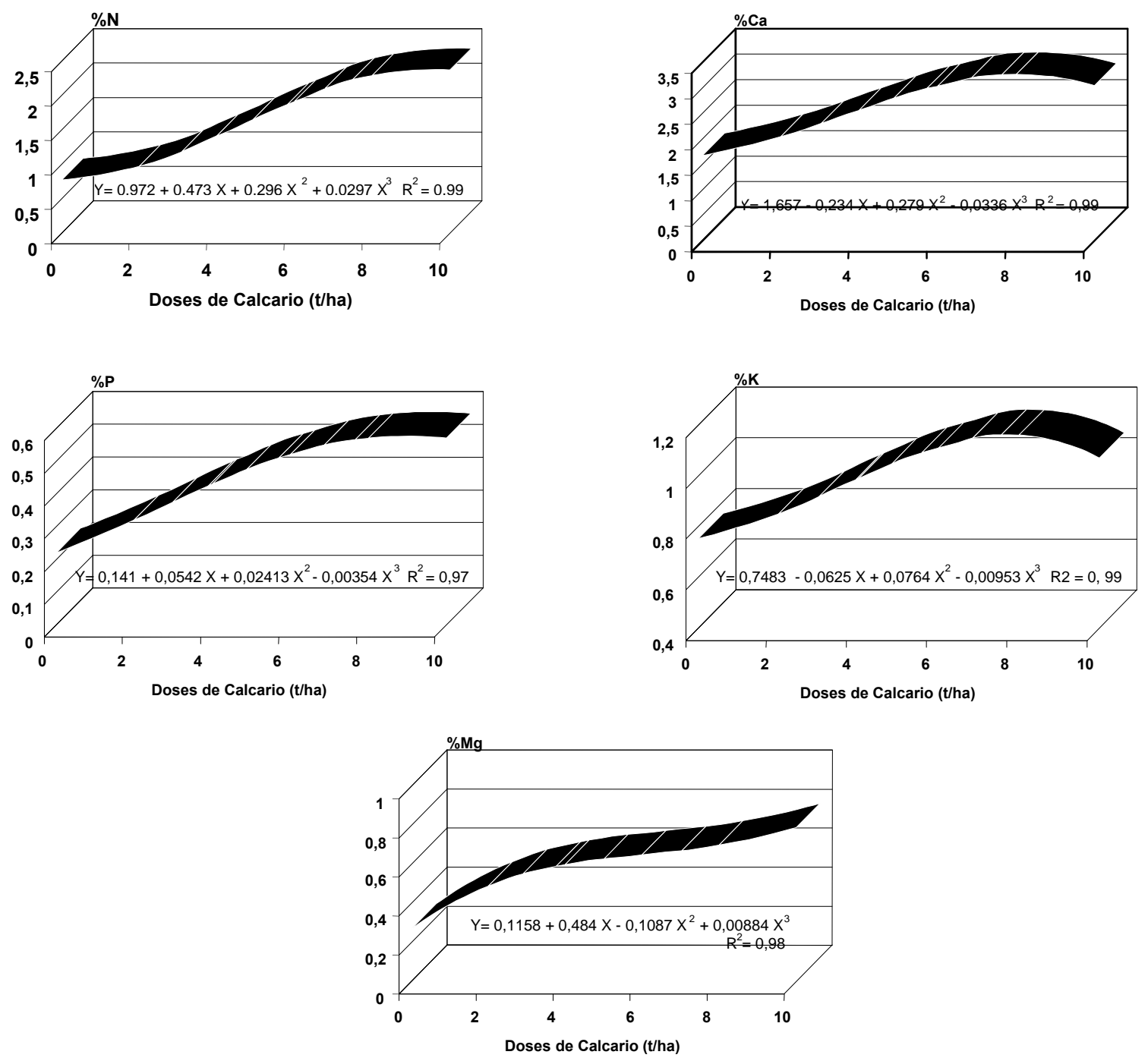

FIGURA 2. Efeito das doses crescentes de calcário sobre os teores de nutrientes nas diferentes partes das plantas de S. obtusifolia aos 56 dias após a emergência. 
TABELA 6. Efeito das doses crescentes de calcário nos acúmulos de nutrientes nas plantas de $S$. obtusifolia aos 56 dias após a emergência.

\begin{tabular}{cccccc}
\hline $\begin{array}{c}\text { Doses } \\
(\mathrm{t} / \mathrm{ha})\end{array}$ & $\mathrm{N}$ & $\mathrm{P}$ & $\begin{array}{c}\mathrm{K} \\
(\mathrm{mg} / \mathrm{planta})\end{array}$ & $\mathrm{Ca}$ & $\mathrm{Mg}$ \\
\hline & & & & & \\
0 & $106.51 \mathrm{~b}^{(1)}$ & $24.62 \mathrm{~b}$ & $81.33 \mathrm{~b}$ & $176.85 \mathrm{bc}$ & $30.47 \mathrm{~b}$ \\
2 & $106.95 \mathrm{~b}$ & $32.52 \mathrm{a}$ & $99.81 \mathrm{a}$ & $248.76 \mathrm{a}$ & $52.64 \mathrm{a}$ \\
4 & $97.69 \mathrm{~b}$ & $31.39 \mathrm{a}$ & $65.78 \mathrm{~d}$ & $167.14 \mathrm{c}$ & $42.05 \mathrm{ab}$ \\
6 & $138.78 \mathrm{a}$ & $36.35 \mathrm{a}$ & $80.33 \mathrm{bc}$ & $211.38 \mathrm{~b}$ & $47.36 \mathrm{a}$ \\
8 & $139.77 \mathrm{a}$ & $35.17 \mathrm{a}$ & $71.99 \mathrm{bcd}$ & $211.82 \mathrm{~b}$ & $41.99 \mathrm{ab}$ \\
10 & $151.44 \mathrm{a}$ & $36.56 \mathrm{a}$ & $67.66 \mathrm{~cd}$ & $190.59 \mathrm{c}$ & $50.80 \mathrm{a}$ \\
\hline F. & $20.92^{* *}$ & $13.64^{* *}$ & $17.09^{* *}$ & $13.61 * *$ & $8.98^{* *}$ \\
D.M.S. & 22.03 & 5.48 & 13.63 & 35.95 & 12.07 \\
C.V. & 7.93 & 7.44 & 7.79 & 7.95 & 12.13 \\
\hline
\end{tabular}

${ }^{* *}$ Significativo ao nível de $1 \%$ de probabilidade.

${ }^{1}$ Médias seguidas da mesma letra não diferem entre si pelo Teste de Tukey ao nível de 5\% de probabilidade.

Utilizando-se a interpretação proposta por Jarrell \& Beverly (1981), nota-se que ocorreu um efeito de concentração em todos os elementos com a elevação das doses de calcário. Entretanto, esta concentração para os macronutrientes $\mathrm{K}, \mathrm{Ca}$ e $\mathrm{Mg}$ foi devida à diminuição do peso seco nas plantas, enquanto que para o $\mathrm{N}$ e $\mathrm{P}$ foi consequiência também da maior absorção destes elementos, uma vez que os acúmulos destes não decresceram com o incremento da calagem, enquanto sua concentração aumentava nos tecidos.

Uma vez que é da solução do solo que as plantas recrutam os nutrientes essenciais para o seu crescimento, as aplicações de corretivos e fertilizantes provocam, na grande maioria das vezes, modificações na composição, solubilidade, disponibilidade e acúmulo de íons, que serão refletidas em respostas fisiológicas diferentes nas plantas, dependendo da espécie e cultivar. Desta maneira, na tentativa de determinar os fatores de maior influência na redução do peso seco de plantas, quando foi elevada a dose de calcário de 2 t/ha para 4 t/ha, serão analisadas as características químicas do solo correspondente a esses tratamentos.

Considerando diversos trabalhos científicos, onde demonstra-se a versatilidade de $S$. obtusifolia de crescer em amplitudes de $\mathrm{pH}$ do solo de 3,2 até 7,9 (Creel et al., 1968; Buchanan et al., 1975 e Murray et al., 1976), o mesmo, no presente trabalho pode ser desconsiderado como fator predominante na redução do peso seco. Esta espécie daninha é frequentemente encontrada em solos originalmente sob vegetação de cerrado, caraterizados por apresentarem elevada acidez, baixa fertilidade e alto poder tampão. É importante destacar que as sementes utilizadas no presente trabalho são provindas de regiões de cerrado nativo do Mato Grosso do Sul.

A elevação da dose de 2 para 4 t/ha de calcário promoveu incrementos no solo de $80 \%$ nos teores de $\mathrm{Mg}$ e $\mathrm{Ca}$ enquanto o teor de $\mathrm{K}$ permaneceu constante. Isto leva a relacionar a redução no acúmulo de matéria seca de $S$. obtusifolia a possíveis efeitos que estes aumentos podem ter ocasionado no equilíbrio iônico no solo e, em consequiência, na disponibilidade de alguns nutrientes. Marmé (1983) cita que concentrações elevadas de Ca na solução do solo podem afetar a absorção de cátions e $\mathrm{Zn}^{2+}$ e aumentar a absorção de ânions e $\mathrm{H}_{2} \mathrm{PO}_{4}$, devido ao acesso desses íons aos sítios de absorção. Menguel \& Kirby (1987) explicam que esta inibição na absorção de um cátion por outro ocorre quando um deles, presente em maior quantidade 
na solução, neutraliza mais rapidamente os equivalentes aniônicos no plasmalema, reduzindo assim a atração eletrostática para as demais espécies catiônicas. Mortvedt \& Khasweth (1986) observaram reduções na absorção de $\mathrm{K}$ em plantas de sorgo em função do aumento da calagem, sendo substituído nos tecidos primeiramente pelo $\mathrm{Mg}$ e em menor grau pelo $\mathrm{Ca}$, em altas taxas de calcário. Quando comparam-se os teores de K verificados nas plantas de S. obtusifolia a outras plantas daninhas (Sander et al., 1981; Weaver \&Hamill, 1985 e Quasem, 1992), e mesmo aqueles verificados por Erasmo et al. (1995) para esta planta, observa-se que os valores são muito baixos.

Vitti (1984) cita que, por serem os cátions, $\mathrm{Ca}, \mathrm{Mg}$ e $\mathrm{K}$ a maior parte dos elementos essenciais encontrados nas cinzas das plantas, a habilidade das mesmas em obter quantidades suficientes para seu ótimo crescimento depende, além do teor absoluto destes elementos no solo, das suas relações
$\mathrm{Ca} / \mathrm{Mg}, \mathrm{K} / \mathrm{Mg}$ e $\mathrm{K} / \mathrm{Ca}$.

A participação do $\mathrm{Ca}$ e $\mathrm{Mg}$ na porcentagem de saturação de bases aumentou em 53 e $47 \%$ quando comparados os tratamentos de 2 e 4 t/ha de calcário, o que provocou a elevação dos valores nas relações $\mathrm{Ca} / \mathrm{K}$ e $\mathrm{Mg} / \mathrm{K}$ e decréscimos na relação $\mathrm{Ca} / \mathrm{Mg}$ (Tabela 4). Silva (1980) constatou decréscimos significativos no peso seco de raízes e parte aérea em plantas de milho, quando a relação cálcio/Mg reduziu de 3:1 para 1:1, fato verificado no presente experimento na dose de 4 t/ha para 6 t/ha de calcário. Mortvedt \& Khaswneh (1986) explicaram reduções no acúmulo de matéria seca em plantas de milho, em resposta a aumento de doses de calcário, a decréscimos nas relações $\mathrm{K} /(\mathrm{Ca}+\mathrm{Mg})$. Nos resultados ora obtidos, este fato foi evidente com o aumento das doses de calcário (Tabela 4).

Procurando-se apoiar a hipótese anterior elaboraram-se algumas correlações entre os teores dos elementos no substrato e o acúmulo de matéria seca nas plantas (Tabela 7).

TABELA 7. Coeficientes de correlação entre a concentração de alguns nutrientes e suas relações e, o acúmulo de matéria seca da parte aérea de S. obtusifolia .

\begin{tabular}{|c|c|}
\hline & $\begin{array}{c}\text { Matéria seca } \\
\mathrm{R}\end{array}$ \\
\hline \multicolumn{2}{|l|}{ Solo } \\
\hline $\mathrm{Ca}$ & $-0.91 * *$ \\
\hline K & $-0.69 * *$ \\
\hline $\mathrm{Mg}$ & $-0.77 * *$ \\
\hline $\mathrm{P}$ & $-0.87 * *$ \\
\hline $\mathrm{Ca} / \mathrm{Mg}$ & $0.61 * *$ \\
\hline $\mathrm{Ca} / \mathrm{K}$ & $-0.79 * *$ \\
\hline $\mathrm{Mg} / \mathrm{K}$ & $-0.68^{* *}$ \\
\hline \multicolumn{2}{|l|}{ Planta } \\
\hline $\mathrm{Ca} / \mathrm{Mg}$ & $0.56^{* * *}$ \\
\hline $\mathrm{Ca} / \mathrm{K}$ & $-0.76^{* *}$ \\
\hline $\mathrm{Mg} / \mathrm{K}$ & $-0.72^{* *}$ \\
\hline
\end{tabular}

Significativo ao nível de $1 \%$ de probabilidade pelo teste t.

Dentre os elementos, o maior índice de correlação foi observado para o Ca, sendo uma relação inversa, com a redução da biomassa acumulada, à medida que houve aumento no teor do nutriente. No entanto, as correlações foram significativas com todos os demais elementos, tornando muito difícil determinar se as relações são diretas ou indiretas. A influência negativa do $\mathrm{Ca}$ de maneira mais expressiva (valores mais elevados de R), pode estar vinculado à relação 
$\mathrm{Ca} / \mathrm{K}$ do substrato, que modificou em função da dose de corretivo atingindo valores considerados críticos para o desenvolvimento normal das plantas, segundo relações já constatadas por diversos autores (Rosolem et al., 1984; Mascarenhas et al., 1988 e Castro \& Meneghelli, 1989). As relações $\mathrm{Ca} / \mathrm{K}$ e $\mathrm{Mg} / \mathrm{K}$ tanto no solo como na planta se correlacionaram negativamente com a matéria seca de plantas, reforçando a provável influência do desequilíbrio entre esses nutrientes no crescimento da planta.

Mesmo não sendo verificado visualmente sintomas de deficiência de micronutrientes, não se pode descartar a possibilidade da redução no peso seco de plantas, como uma função do nível crítico de algum micronutrientes à elevação do ph do solo.

\section{LITERATURA CITADA}

ANDREASEN, C., STREIBIG, J. C., HASS, H. Soil properties affecting the distribution of 37 weed species in Danish field. Weed Res., v. 31, p. 181-187, 1991.

BUCHANAN, G. .A., HOVELAND, C.S., HARRIS, M.C. Response of weed to soil pH. Weed Sci., v.23, n.6, p.473-7, 1975.

CASTRO, A.F. de, MENEGHELLI, N. do A. As relações $\mathrm{K}^{+} /\left(\mathrm{Ca}^{++} \mathrm{Mg}^{++}\right)^{1 / 2}$ e no $\mathrm{K}^{+} /\left(\mathrm{Ca}^{++} \mathrm{Mg}^{++}\right)$solo e as respostas a adubação potássica. Pesqui. Agropecu. Bras., Brasília, v. 24 n.6, p. 751-60, 1989.

CREEL, J.M., HOVELAND JR, C.S., BUCHANAN, G.A. Germination, growth and ecology of sicklepod. Weeds, v.16, p.396-400, 1968.

ERASMO, E. A. L. Crescimento, nutrição mineral e respostas a calagem em Senna obtusifolia (L.) IRWIN \& Barneby. Jaboticabal, 1995. 88p. Tese (Doutorado) Faculdade de Ciências Agrárias e Veterinárias, Universidade Estadual
Paulista "Julio Mesquita Filho".

HOAGLAND, D.R., ARNON, D.J. The water culture method of growing plants without soil. Berkeley: University of California, 1950.

JARRELL, W.M., BEVERLY, R.B. The dilution effects in plant nutrition studies. Adv. Agron., v. 34, p. 197-224, 1981.

MARME, D. Calcium transport and function. In: LAUCHLI, A.,BIELZSKI, R.L., Inorganc plant nutrition. Berlin: Springerverlog, 1983. p. 599-619.

MASCARENHAS, H. A. A., et al. Deficiência de potássio em soja no Estado de São Paulo: melhor entendimento do problema $\mathrm{e}$ possíveis soluções. Piracicaba, POTAFOS, 1988. $12 \mathrm{p}$.

MENGUEL, K. \& KIRKBY, E. A. Principles of plant nutritions. 4 ed. Bern, Internacional Potash. Institute, 1987. p. 444.

MORTVEDT, J. J. \& KHASAWNEH, F. E. Effects of growth responses on cationic relationships in plants. Soil Sci., v. 141, n. 3, p. 200-207, 1986.

MURRAY, D. S., THURLOW, D. L., BUCHANAM, G. .A. Sickelepod in the southeast. Weeds, v.4, p. 17-8, 1976.

PAVANI, M. C. D. Estudos sobre o crescimento, nutrição mineral de Cenchrus echinatus L. e respostas a diferentes níveis de fósforo, $\mathrm{pH}$ e calagem. Jaboticabal, 1992, 110 p.,Tese (Doutorado em Agronomia) - Faculdade de Ciências Agrárias e Veterinárias de Jaboticabal, Universidade Estadual Paulista.

QUASEM, J. R. Nutrient accumulation by weeds and associated vegetable crops. J. Hortic. Sci., $\quad$ v.62, n.2, p.189-195, 1992. 
RAMAKRISHNAN, P. S. \& GUPTA, V. Nitrogen, phosphorus and potassium nutrition of the edaphic ecotypes in Cynodon dactylon (L.) Pers. Ann. Bot., v.37, n.152, p.885-94, 1973.

ROSOLEM, C. A., MACHADO, J. R., BRINHOLI, O. Efeito das relações $\mathrm{Ca} / \mathrm{Mg}$, $\mathrm{Ca} / \mathrm{K}$ e $\mathrm{Mg} / \mathrm{K}$ do solo na produção de sorgo sacarino. Pes. Agropec. Bras., Brasília, v.19, n.12, p.1443-48, 1984.

SANDER, D. C., GRAYSON, A. S., MONACO, T. J. Mineral content of tomato (Licopersicon esculentum) and four competing weed species. Weed Sci., v.29, n.5, p.590-593, 1981.

SILVA, J. E. da. Balanço de cálcio e magnésio e desenvolvimento do milho em solos sob cerrado. Pes. Agropec. Bras., v.15, n.3, p.329-333, 1980.

SARRUGE, J.R. \& HAAG, H. P. Análises químicas em plantas. Piracicaba: ESALQ/USP, 1974. 56p. (mimeogr.).

TEEM, D. H., HOVELAND, C. S., BUCHANAN, G.A. Primary root elongation of three weed species. Weed Sci., v.22, n.1, p.47-50, 1974.

VITTI, G. C. Acidez e calagem. Corderópolis: Estação experimental de Limeira: SP, 1984, p. 1-19 (palestra).

WEAVER, S. E. \& HAMILL, A. S. Effects of soil $\mathrm{pH}$ on competitive ability and leaf nutrient content of corn (Zea mays L.) and three weed species. Weed Sci., v.33, n.4, p.447-451, 1985. 